\title{
SIMULAÇÃO DE COMPOSIÇÃO FERROVIÁRIA ACIONADA POR MOTORES DE INDUÇÃO E INVERSORES DE TENSÃO
}

\author{
Cassiano Lobo Pires \\ Silvio Ikuyo Nabeta \\ José Roberto Cardoso \\ LMAG - Laboratório de Eletromagnetismo Aplicado \\ PEA - Departamento de Engenharia de Energia e Automação Elétricas \\ Escola Politécnica da Universidade de São Paulo \\ Av. Prof. Luciano Gualberto, tr.3, nº 158 CEP 05508-900 São Paulo, SP \\ email: cassiano@pea.usp.br
}

\begin{abstract}
This paper presents a study of train performance simulation for inverter-controlled asynchronous drives in DC railways. This type of equipment is new in Brazilian railway and subway systems and the need to model and to simulate became an important issue and the aim of this paper. The development of the model is based on electric railway traction theory for train dynamics, on inverter-fed induction machine control and induction machine theory to calculate the input line current from power supply, the most important result. As an example, a modeling and simulation of one train is shown. The analysis of the results shows that the input line current from power supply may be obtained by a simplified model. This hypothesis is studied, the results are compared and the simplified model is then validated.
\end{abstract}

KEYWORDS: Electric railway traction, train performance simulation

\section{RESUMO}

O presente trabalho traz o estudo da simulação do desempenho (simulação de marcha) de uma composição ferroviária acionada através de inversores de tensão e

Artigo Submetido em 25/04/02

1a. Revisão em 07/04/03;

2a. Revisão em 10/09/04

Aceito sob recomendação do Ed.Assoc.Prof. José Antenor Pomilio motores de indução e alimentada em corrente contínua, um tipo de material rodante recentemente introduzido no Brasil. Tal fato faz com que seja necessário o desenvolvimento de métodos de análise das características deste tipo de material, objetivo maior deste trabalho. A metodologia de análise aqui desenvolvida tem como base a teoria da tração elétrica para o equacionamento da dinâmica do movimento, a teoria do controle do motor de indução e a teoria do funcionamento do próprio motor de indução para a obtenção da corrente captada por esta composição, o principal resultado obtido. A aplicação desta metodologia é dada através da simulação de uma composição em um trecho ferroviário. Uma análise posterior dos resultados estudados traz a possibilidade de uma simplificação do método de obtenção da corrente captada. Tal possibilidade é apresentada e comparada com os dados anteriormente obtidos validando-a.

PALAVRAS-CHAVE: Tração elétrica, simulação ferroviária

\section{LISTA DE SÍMBOLOS}

$\begin{array}{ll}b & \text { bitola (distância entre trilhos) }[\mathrm{m}] \\ F_{a d} & \text { força de aderência }[\mathrm{kN}] \\ F_{\text {motor }} & \text { esforço motor }[\mathrm{kN}] \\ f & \text { frequência da fonte }[\mathrm{Hz}] \\ g & \text { aceleração da gravidade }\left[\mathrm{m} / \mathrm{s}^{2}\right] \\ I_{d} & \text { corrente contínua captada pelo trem }[\mathrm{A}] \\ I_{M} & \text { componente de magnetização da corrente } \\ & \text { por fase do estator (valor eficaz) }[\mathrm{A}] \\ I_{R} & \text { componente de carga da corrente por } \\ & \text { fase do estator (valor eficaz) [A] }\end{array}$




\begin{tabular}{|c|c|}
\hline$I_{S}$ & corrente por fase do estator (valor eficaz) \\
\hline & inclinacão da rampa [\%o] \\
\hline isa,isb,isc & corrente por fase no motor $[\mathrm{A}]$ \\
\hline$k_{G}$ & razão de transmissão [1] \\
\hline$k_{n}$ & $(n=1,2,3$ e 4$)$ constantes \\
\hline$L_{M}$ & indutância de magnetização por fase $[\mathrm{H}]$ \\
\hline$L_{R}$ & $\begin{array}{l}\text { indutância de dispersão do rotor por fase } \\
{[\mathrm{H}]}\end{array}$ \\
\hline$L_{S}$ & $\begin{array}{l}\text { indutância de dispersão do estator por } \\
\text { fase }[\mathrm{H}]\end{array}$ \\
\hline$m$ & massa do trem $[\mathrm{t}]$ \\
\hline$n_{m}$ & número de motores [1] \\
\hline$n_{r m}$ & velocidade de rotação do motor [rpm] \\
\hline$P_{a d}$ & peso aderente do trem $[\mathrm{kN}]$ \\
\hline$P_{\text {in }}$ & $\begin{array}{l}\text { potência de entrada do motor (por fase) } \\
{[\mathrm{kW}]}\end{array}$ \\
\hline$P_{\text {motor }}$ & $\begin{array}{l}\text { potência desenvolvida pelo eixo do } \\
\text { motor }[\mathrm{kW}]\end{array}$ \\
\hline$P_{U}$ & potência desenvolvida pelo trem [kW] \\
\hline$R_{C}$ & resistência devido às curvas $[\mathrm{kN}]$ \\
\hline$R_{i}$ & resistência devido às rampas $[\mathrm{kN}]$ \\
\hline$R_{M P T}$ & resistência normal do trem [kN] \\
\hline$R_{R}$ & resistência por fase do rotor $[\Omega]$ \\
\hline$R_{S}$ & resistência por fase do estator $[\Omega]$ \\
\hline$r_{e}$ & raio da roda $[\mathrm{m}]$ \\
\hline$s$ & escorregamento $[\%]$ \\
\hline$T_{\text {motor }}$ & torque do motor $[\mathrm{kN} \cdot \mathrm{m}]$ \\
\hline$t$ & tempo $[\mathrm{s}]$ \\
\hline$v$ & velocidade do trem $[\mathrm{m} / \mathrm{s}]$ \\
\hline$U_{d}$ & tensão na catenária ou terceiro trilho $[\mathrm{V}]$ \\
\hline$U_{S}$ & $\begin{array}{l}\text { tensão eficaz por fase nos terminais do } \\
\text { motor [V] }\end{array}$ \\
\hline usa, usb,usc & $\begin{array}{l}\text { tensão por fase nos terminais do motor } \\
{[\mathrm{V}]}\end{array}$ \\
\hline$\Phi_{A G}$ & fluxo no entreferro [Wb] \\
\hline$\varphi$ & ângulo do fator de potência $\left[^{\circ}\right]$ \\
\hline$\eta_{m}$ & rendimento do motor [1] \\
\hline$\eta_{T}$ & rendimento da transmissão [1] \\
\hline$\mu$ & coeficiente de aderência [1] \\
\hline$\rho$ & raio de curvatura $[\mathrm{m}]$ \\
\hline
\end{tabular}

\section{INTRODUÇÃO}

As vantagens oferecidas pelo motor de indução trifásico quando utilizados na tração elétrica já eram conhecidas desde o final do século XIX porém, seu emprego não foi amplo pela falta de um equipamento de controle adequado.

O desenvolvimento no início dos anos oitenta do tiristor tipo GTO de alta potência e a combinação com controle microprocessado, se tornou o caminho para a aplicação do motor de indução na construção de locomotivas e de TUEs (trem unidade elétrico). Esta aplicação começou a ser feita de forma ampla na Europa a partir da metade da década de oitenta. Dez anos mais tarde, o IGBT começou a substituir os GTOs nos conversores auxiliares das locomotivas e acionamentos principais em veículos leves.

No Brasil, prevaleceram os acionamentos através de motores de corrente contínua e recortadores (choppers). Foi somente no ano de 2000, com a modernização do material rodante das linhas de trens metropolitanos em São Paulo sob o controle da CPTM (Companhia Paulista de Trens Metropolitanos) e a construção da Linha 5 do Metrô de São Paulo é que composições acionadas por inversores e motores de indução começaram a ser empregadas.

A utilização desta nova tecnologia no âmbito nacional traz a necessidade de novos estudos, modelos de simulação e metodologias de análise tanto para o dimensionamento de novas linhas quanto para os estudos de viabilidade de aplicação desta nova tecnologia em linhas já existentes.

O presente trabalho tem como objeto de estudo a modelagem de uma composição ferroviária compreendendo os aspectos dinâmicos do seu movimento entre as estações e seu reflexo na corrente elétrica consumida (simulação de marcha). Para ilustrar o artigo, o cálculo do desempenho de uma composição em um dado percurso é feito sendo a locomotiva alimentada em corrente contínua e acionada por inversores e motores de indução tipo gaiola.

\section{VANTAGENS DO MOTOR DE INDUÇÃO PARA A TRAÇÃO ELÉTRICA}

Os tradicionais motores de corrente contínua com excitação em série utilizados em tração elétrica possuem alguns itens vulneráveis como escovas e comutadores. Em contraposição, o motor de indução tipo gaiola é um motor robusto e de extrema simplicidade em sua construção além de não possuir conexões externas com o rotor.

A principal vantagem do motor de indução tipo gaiola sobre os tradicionais motores dotados de comutador é justamente a ausência deste. Motores com comutadores limitam sua capacidade de fornecer potência nominal em altas velocidades por causa do faiscamento excessivo do comutador causado, entre outros fatores, pelas tensões de reatância. Este fato ocorre também na freagem quando a potência é igualmente limitada (Largiadèr, 1970).

A relação peso / potência e o momento de inércia do motor de indução tipo gaiola são menores do que nos motores de corrente contínua. Isto significa mais potência para o mesmo volume ou menos peso para a mesma potência. Desta forma, ele pode ser posicionado no espaço confinado do truque que pode por sua vez ser mais leve, causando menos danos à via. 
Quanto à parte de custos, levando em conta apenas o motor de tração, o custo inicial é baixo devido a uma redução de material do motor e a ausência de comutadores, normalmente caros. Os custos operacionais são reduzidos uma vez que não existem escovas para serem trocadas e comutadores para serem retificados.

\section{DINÂMICA DO MOVIMENTO}

A simulação de marcha constitui uma ferramenta poderosa no dimensionamento e operação de um sistema de tração elétrica. Isto se deve ao fato da simulação de marcha fornecer como resultado a posição de um trem na via e em função desta posição, o tempo de percurso, a velocidade e a corrente absorvida, entre outros parâmetros.

Para o cálculo de tais parâmetros, é tomada uma das variáveis da cinemática como independente (espaço percorrido ou tempo de percurso) e através de integrações sucessivas, determinam-se as demais.

O movimento de um trem, definido pelo traçado da via, é similar ao de um ponto material. Tal movimento é descrito pela Segunda Lei de Newton que pode ser expressa na forma diferencial:

$$
F_{\text {motor }}-\left(R_{M P T}+R_{C} \pm R_{i}\right)=m \cdot 1,1 \cdot \frac{d v}{d t}
$$

no qual $F_{\text {motor }}$ é a força linear produzida pelos motores de tração. $O$ fator de 1,1 é um coeficiente que leva em conta a inércia das massas girantes (eixos montados e rotores).

\subsection{Esforços resistentes}

A força que se opõe ao movimento do trem pode ser dividida em duas partes:

\subsubsection{Resistências normais}

As resistências normais $R_{M P T}$ são as resistências de toda a natureza, que em reta e nível, se opõe ao movimento do trem. São dadas por fórmulas empíricas que foram desenvolvidas ao longo do tempo por diversos autores. Dentre estas fórmulas empíricas, destacam-se as desenvolvidas por W. J. Davis Jr. (1926) pois são ainda utilizadas no Brasil e nos Estados Unidos para quantificar as resistências normais do trem (Toledo et alii, 1987).

Estas fórmulas empíricas são sempre um trinômio em função da velocidade. $O$ termo constante em relação à velocidade, compreende a resistência do rolamento dos aros das rodas sobre os trilhos e o atrito nos mancais do eixo. $\mathrm{O}$ termo diretamente proporcional à velocidade é devido principalmente à resistência de atrito do friso das rodas com os trilhos e também devido ao atrito causado pelas oscilações e balanços. Por fim, o termo dependente do quadrado da velocidade representa a parte aerodinâmica sendo sensível a diversos fatores como a forma frontal da composição, intervalo entre trens e saliências na estrutura (Toledo et alii, 1987; Kaller e Allenbach, 1995; Filipović, 1995; Courtois et alii, 1998).

\subsubsection{Resistências acidentais}

As resistências acidentais são classificadas em dois tipos: resistências devido às curvas e resistências devido às rampas.

\subsubsection{Resistências devido às curvas}

A resistência acidental devido às curvas não pode ser avaliada teoricamente, nem medida separadamente. Ela é estabelecida também através de fórmulas empíricas que dão o valor da resistência $R_{C}$ encontrada durante a circulação da composição em uma curva. Uma expressão bastante utilizada é a de Desdouits (Toledo et alii, 1987):

$$
R_{C}=\frac{4,9 \cdot b}{\rho} \cdot m
$$

\subsubsection{Resistências devido às rampas}

A resistência devido à gravidade $R_{i}$ é decorrente dos aclives e declives ao longo do percurso. Assim, uma composição que se encontra em uma rampa pode ter o seu peso $(m . g)$ decomposto em um componente normal (função do coseno do ângulo de inclinação da rampa) e em um outro tangencial (função do seno do ângulo de inclinação da rampa) à rampa.

O componente tangencial à rampa é a resistência devido às rampas. Contudo, para o caso da tração metroferroviária, o seno do ângulo de inclinação da rampa é aproximadamente igual à tangente deste mesmo ângulo. Este fato é bastante conveniente uma vez que as rampas metroferroviárias não são dadas em função do seu ângulo, mas sim em função da tangente deste ângulo, representado por $i$. A seguinte expressão dá o valor da resistência devido às rampas (Toledo et alii, 1987; Allenbach e Kaller, 1995; Filipović, 1995; Courtois et alii, 1998).

$$
R_{i}=m \cdot g \cdot i \cdot 10^{-3}
$$

É importante notar que os aclives introduzem uma resistência contra o movimento do trem enquanto que os declives atuam a favor do movimento, sendo este o único caso de resistência negativa em tração.

\subsection{Força de aderência}

O esforço motor, que é transferido da roda para o trilho, é limitado pela força de aderência. Esta força, em uma 
primeira aproximação, corresponde à força de atrito estática.

Se o esforço motor, durante o regime de tração, for maior que o módulo da força de aderência, as rodas começam a centrifugar isto é, a velocidade periférica da roda torna-se maior que a velocidade do trem (patinagem). Do mesmo modo, se este limite for superado durante o regime de freagem, as rodas começam a deslizar isto é, a velocidade periférica da roda torna-se menor que a velocidade do trem aumentando a distância de freagem, o que acarreta em uma série de riscos (Filipović, 1995; Courtois et alii, 1998). Assim,

$$
\left|F_{\text {motor }}\right| \leq\left|F_{a d}\right|
$$

Como a força de aderência limita o esforço motor, a expressão para esta força leva em conta apenas os eixos motores e consequentemente, apenas veículos motorizados de um trem. O peso total sobre os eixos motores é chamado de peso aderente. Deste modo, a força de aderência é dada por:

$$
\left|F_{a d}\right|=P_{a d} \cdot \mu
$$

Os valores do coeficiente de aderência $\mu$ para as diversas velocidades são fornecidos através de ensaios nos quais se determinam os esforços de tração imediatamente anteriores à patinagem. Um ensaio bastante difundido foi feito por E. W. Curtius e A. Kniffler em 1943. A expressão resultante deste ensaio é dada por:

$$
\mu=0,33 \cdot \frac{8+0,1 \cdot(3,6 \cdot v)}{8+0,2 \cdot(3,6 \cdot v)}
$$

Para os veículos de tração mais modernos esta expressão fornece valores mínimos com distância suficiente dos valores de pico, oferecendo segurança (Allenbach e Kaller, 1995; Filipović, 1995).

\subsection{Variáveis do movimento referidas ao motor}

De um modo geral, o motor de tração transmite seu torque e sua velocidade de rotação através de um sistema de engrenagens para o eixo motorizado do truque e consequentemente, para a roda do trem. Este conjunto de engrenagens possui uma razão de transmissão e uma eficiência.

O cálculo do torque desenvolvido por cada um dos $n_{m}$ motores de tração pode ser feito a partir do esforço motor $F_{\text {motor }}$ desenvolvido pelo trem. Se for considerada uma distribuição igual do esforço motor entre esses motores e também que as rodas possuem um mesmo raio igual $r_{e}$, é válida a seguinte expressão:

$$
T_{\text {motor }}=\frac{F_{\text {motor }} \cdot r_{e} \cdot k_{G}}{n_{m} \cdot \eta_{T}}
$$

E a velocidade de rotação motor pode ser expressa através da velocidade do trem por:

$$
n_{r m}=\frac{60 \cdot v}{2 \cdot \pi \cdot r_{e} \cdot k_{G}}
$$

A potência desenvolvida pelo trem e a potência mecânica no eixo do motor se relacionam através de:

$$
P_{\text {motor }}=\frac{P_{U}}{n_{m} \cdot \eta_{T}}
$$

\section{MODELO ELÉTRICO DO SISTEMA DE TRAÇÃO}

\subsection{Circuito equivalente}

O modelo de circuito elétrico utilizado para o motor de indução é o dado em Fitzgerald, Kingsley e Kusko (1975) e Mohan, Undeland e Robbins (1995). Apenas salientando alguns aspectos do circuito equivalente por fase, a corrente do estator, descontando as perdas no ferro segundo a orientação adotada em Fitzgerald, Kingsley e Kusko (1975), pode ser decomposta em duas partes: um componente de magnetização e um componente de carga.

O componente de carga produz uma força magnetomotriz que cancela a força magnetomotriz real do rotor. $\mathrm{O}$ componente de magnetização é a corrente de estator necessária para se criar o fluxo resultante no entreferro. Deste modo,

$$
I_{M}=k_{1} \cdot \Phi_{A G}
$$

Esta corrente está atrasada de $90^{\circ}$ em relação à força contraeletromotriz do estator e pode ser considerada como uma corrente atravessando um ramo paralelo composto por $L_{M}$, conforme a Figura 1.

$\mathrm{O}$ efeito sobre as características elétricas da máquina dos fluxos de dispersão do estator e do rotor, que induzem tensões nos próprios enrolamentos, podem ser levados em conta através das indutâncias de dispersão do estator e do rotor. Em série com $L_{S}$ pode ser posta uma resistência que representa a queda ôhmica deste enrolamento. Uma resistência rotórica, em série com $L_{R}$, que depende do escorregamento, também pode ser posta. Tal resistência pode ser decomposta em duas partes: uma que representa a queda ôhmica nas barras do rotor e outra que representa a potência mecânica do motor por fase. Um esquema do circuito equivalente por fase utilizado é dado na Figura 1. 


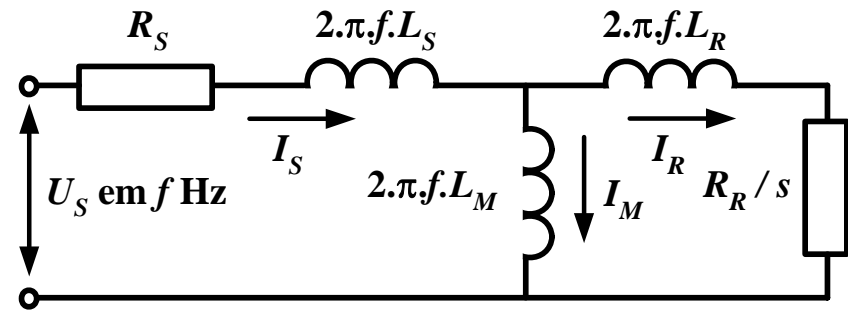

Figura 1 - Circuito equivalente por fase utilizado.

Vale ressaltar também que o torque motor desenvolvido é proporcional ao produto das amplitudes das forças magnetomotrizes do rotor e do estator. Sua expressão pode ser dada em função da força magnetomotriz do rotor, do ângulo entre a força magnetomotriz do estator e a força magnetomotriz resultante entre as forças magnetomotrizes do estator e do rotor (ângulo de carga) e também do fluxo $\Phi_{A G}$ produzido pelo efeito combinado das correntes do estator e do rotor.

Uma simplificação adotada é que o ângulo de carga se mantém em seu valor ótimo de $90^{\circ}$ considerando que (2.f. $\left.L_{R}\right)<<R_{R}$. Outra simplificação é assumir que a tensão $U_{S}$ é aproximadamente igual à força contra-eletromotriz induzida para freqüências não muito baixas dado que a queda de tensão no enrolamento do estator é pequena.

Assim, a corrente $I_{R}$ pode ser dada através do circuito equivalente por (Mohan, Undeland e Robbins, 1995):

$$
I_{R} \cong k_{2} \cdot \Phi_{A G} \cdot f \cdot s
$$

As correntes do circuito equivalente podem ser relacionadas do seguinte modo (Mohan, Undeland e Robbins, 1995):

$$
I_{S} \cong \sqrt{I_{R}^{2}+I_{M}^{2}}
$$

O torque $T_{\text {motor }}$ pode então ser expresso por (Mohan, Undeland e Robbins, 1995):

$$
T_{\text {motor }} \cong k_{3} \cdot \Phi_{A G} \cdot I_{R}
$$

E a tensão $U_{S}$ é dada por (Mohan, Undeland e Robbins, 1995):

$$
U_{S} \cong k_{4} \cdot \Phi_{A G} \cdot f
$$

\subsection{Controle dos motores de indução}

O esquema de controle dos motores de indução é o mesmo observado em Hill (1994), Mohan, Undeland e Robbins (1995), Courtois et alii (1998) e Steimel (1998), onde se distinguem três regiões de controle.

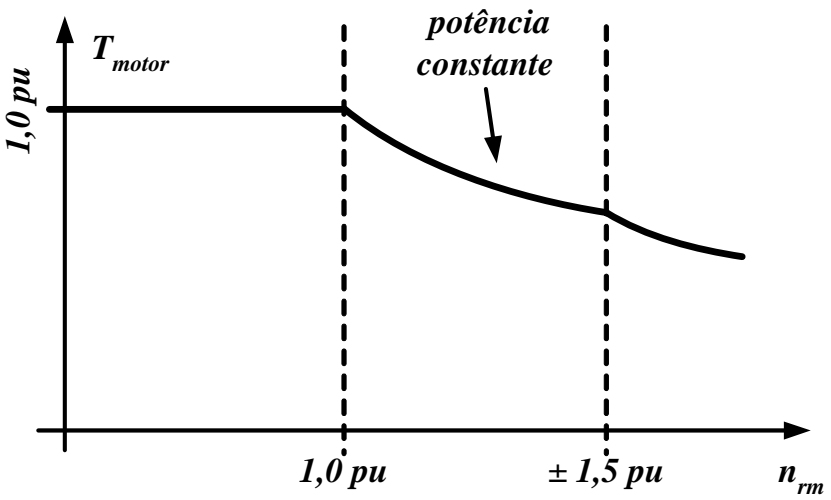

Figura 2 - Representação das três regiões de controle de um motor de indução.

Como se observa na Figura 2, a primeira região é conhecida como região de torque constante. $\mathrm{O}$ torque $T_{\text {motor }}$ é mantido constante e, conseqüentemente, a corrente $I_{R}$, até que a velocidade $n_{r m}$ atinja o seu valor nominal. Para isto a freqüência de escorregamento $(f . s)$ e o fluxo $\Phi_{A G}$ são mantidos constantes. A tensão $U_{S}$ é baixa quando o trem está parado e vai aumentando até atingir o seu valor nominal conforme $f$ e $n_{r m}$ aumentam, lembrando que o fluxo $\Phi_{A G}$ é mantido constante e em seu valor nominal.

Acima da velocidade nominal do motor, os parâmetros são controlados de maneira diferente. A tensão $U_{S}$ é mantida em seu valor nominal. Como a freqüencia $f$ aumenta, o fluxo $\Phi_{A G}$ tem seu valor diminuído de maneira proporcional. Para que a corrente $I_{R}$ continue constante, a freqüência de escorregamento aumenta e deixa de ser constante nesta região. Deste modo, $s$ é mantido constante. $\mathrm{O}$ torque $T_{\text {motor }}$ irá cair conforme a velocidade aumenta porém, a potência $P_{\text {motor }}$ é constante nesta região.

A potência é mantida constante até por volta de 1,5 a 2,0 vezes a velocidade nominal do motor, dependendo da sua construção. O torque $T_{\text {motor }}$ se aproxima de seu valor máximo. A freqüência de escorregamento atinge valores críticos. Assim, esta freqüência é mantida constante conforme $f$ continua a aumentar. A corrente $I_{R}$ e o fluxo $\Phi_{A G}$ diminuem com o aumento de $f$. A tensão $U_{S}$ é mantida constante em seu valor nominal. A potência $P_{\text {motor }}$ nesta terceira região de controle é menor do que na região anterior.

\subsection{Freagem}

A freagem é realizada através da operação da máquina de indução como gerador sendo mecanicamente acionada acima da velocidade síncrona, que é função da freqüência $f$.

O modo de excitação da máquina de indução utilizado, seguindo Mohan, Undeland e Robbins (1995), é através de 
uma fonte externa. As tensões alternadas devem estar presentes nos terminais do estator para que sejam produzidos fluxo e força magnetomotriz no entreferro. A corrente $I_{M}$ é então fornecida através da fonte.

Caso a velocidade $n_{r m}$ seja maior que a velocidade do campo girante produzido pelo estator alimentado por uma tensão de certa freqüência, o escorregamento será negativo.

A corrente induzida no rotor gera uma força magnetomotriz que gira no espaço à velocidade síncrona. Esta força magnetomotriz gira no sentido oposto ao rotor e em interação com a força magnetomotriz do estator. O torque será então resistente e a máquina funcionará como gerador fornecendo potência à fonte.

O circuito equivalente não sofre modificações na operação em freagem. As modificações serão observadas na representação fasorial da máquina de indução.

A tensão $U_{S}$ e a corrente de magnetização $I_{M}$ permanecerão com a mesma fase da operação da máquina como motor e distantes $90^{\circ}$ entre si de acordo com as simplificações anteriormente adotadas. Também de acordo com estas simplificações, a corrente $I_{R}$ irá sofrer uma modificação em sua fase durante a operação como gerador. Seu fasor estará defasado de $180^{\circ}$ do fasor da força contra-eletromotriz induzida. Logo, o fasor da corrente do estator estará defasado de um ângulo maior que $90^{\circ}$ em relação ao fasor da força contra-eletromotriz induzida.

Esta defasagem de mais de $90^{\circ}$ entre os fasores da corrente do estator e da força contra-eletromotriz (e como conseqüência também da tensão $U_{S}$ ) é levada em conta somente sob o ponto de vista analítico.

De acordo com Langsdorf (1981), o componente de potência ativa da corrente $I_{S}$ é considerado em oposição de fase à tensão $U_{S}$ para mostrar que a energia flui do motor para o conversor. O ângulo $\varphi$ (diferença de fase entre $U_{S} \mathrm{e}$ $I_{S}$ ) de um gerador de indução é considerado como o co-seno de $180^{\circ}$ menos o ângulo entre os fasores de tensão e corrente do estator. Em outras palavras, é o ângulo menor que $90^{\circ}$ entre o eixo do fasor de tensão e o eixo do fasor de corrente do estator.

\subsubsection{Freagem regenerativa}

A freagem regenerativa tornou-se de importância maior nos sistemas metroviários e ferroviários urbanos porque o tempo entre composições é pequeno e as partidas e freagens são freqüentes.

Um trem freando pode transferir a energia de freagem tanto para a rede de alimentação quanto para um resistor de freagem. Quando uma composição retorna a sua energia de freagem para a rede de alimentação em corrente contínua (sistema de alimentação comum nos transportes urbanos), esta energia deve ser utilizada por outra composição, ou retornada para a concessionária, caso a regeneração para o lado CA nas subestações retificadoras seja possível.

A característica de esforço motor em função da velocidade na freagem pode possuir duas ou três regiões de controle distintas. Para o caso desta característica ter três regiões, o controle dos motores será exatamente igual ao controle em regime de tração. Porém existe o caso em que a característica de esforço em função da velocidade possui duas regiões: uma de torque constante e outra de potência constante. Tal característica é comum nos trens alimentados em corrente contínua. A terceira região de controle pode ser evitada na freagem através de um dimensionamento apropriado dos resistores de freagem.

A região de potência constante na freagem é controlada igualmente à região de potência constante no regime de tração (Plette e Plunkett, 1977). Para a região de torque constante, a estratégia de controle é similar à da região de torque constante em tração. Vale notar que o fluxo resultante no entreferro nesta região de torque constante é mantido em seu valor nominal, independente da velocidade e da carga.

\subsection{Corrente no pantógrafo / coletor do terceiro trilho}

A corrente captada por um trem em uma linha alimentada em corrente contínua é a mesma corrente do lado CC de um inversor trifásico, considerando este inversor ligado diretamente à rede de alimentação sem recortadores (choppers) intermediários.

A tensão $U_{d}$ é assumida ser totalmente contínua. Vale observar que durante a simulação, seu valor é $90 \%$ da tensão nominal de alimentação durante o regime de tração e $110 \%$ durante o regime de freagem, como indicado em Martins (1986).

Como a freqüência de chaveamento do inversor é muito alta, o filtro no lado CC do inversor irá possuir indutâncias e capacitâncias quase nulas, resultando em um armazenamento nulo de energia. Todos os componentes de alta freqüência devido ao chaveamento são aí filtrados (Mohan, Undeland e Robbins, 1995).

Se for considerado que as tensões usa, usb e usc e as correntes isa, isb e isc são senoidais, trifásicas e equilibradas e também que as correntes são defasadas de $\varphi$ em relação às tensões pode-se equacionar a potência instantânea de entrada e de saída. Assim,

$$
U_{d} \cdot I_{d}=u s a \cdot i s a+u s b \cdot i s b+u s c \cdot i s c
$$


Desenvolvendo a equação (15) pode-se chegar a uma expressão para o valor da corrente $I_{d}$ captada pelo trem. Deste modo,

$$
I_{d}=n_{m} \cdot \frac{3 \cdot U_{S} \cdot I_{S}}{U_{d}} \cdot \cos (\varphi)
$$

\section{ASPECTOS COMPUTATIONAIS}

Para a realização da simulação de marcha através de um computador, a equação (1) deve ser resolvida através de métodos numéricos. Esta resolução, mais o equacionamento do comportamento elétrico de uma composição, resultou em várias rotinas de cálculo escritas para o ambiente computacional MATLAB (Pires, 2002).

A simulação de marcha aqui descrita é composta basicamente de três etapas: entrada de dados, pré-simulação e simulação. Cada uma destas etapas é composta de algumas rotinas de cálculo.

\subsection{Entrada de dados}

A entrada de dados é iniciada com a solicitação dos dados da composição. Primeiramente, deve-se informar quantos veículos este trem possui e qual o tipo (vagão, locomotiva, carro). O cálculo da resistência normal da composição é feito de acordo com as expressões gerais desenvolvidas por W. J. Davis Jr. (1926). Para tanto são necessários, para cada veículo, a massa, o número de eixos e a seção transversal. Como saída armazenada, tem-se a massa total do trem e os coeficientes do trinômio que representam a $R_{M P T}$.

As resistências acidentais são contabilizadas por uma outra rotina que, em primeiro lugar, necessita do fornecimento do comprimento da via e da bitola utilizada. O comprimento da via é então dividido em trechos de perfis que podem ser planos, rampas, curvas ou rampas e curvas combinadas. De acordo como o perfil do trecho são aplicadas a equação (2) ou/e a equação (3). Para isso é necessária a entrada do raio da curva ou/e a inclinação da rampa para cada caso.

Como último dado de entrada tem-se a velocidade máxima permitida por trecho. A entrada dos dados é feita igualmente à entrada do perfil da linha: o comprimento da via é dividido em vários trechos de mesma velocidade máxima permitida.

\subsection{Pré-simulação}

O método mais comum para se encontrar o ponto onde a composição deve iniciar a freagem é através da aplicação de equações provenientes da Mecânica que levam em consideração a velocidade inicial, a velocidade final e uma aceleração constante. Um exemplo deste tipo de equação pode ser encontrado em Martins (1986). O problema da utilização destas equações é que a aceleração não é constante devido às mudanças na geometria da via e também devido às resistências normais que variam com a velocidade do trem, como pode ser observado através da equação (1).

A solução desenvolvida para se encontrar o ponto do início da freagem foi a pré-simulação de marcha feita antes da simulação final. O objetivo principal desta pré-simulação é definir de modo iterativo o ponto entre duas estações onde o trem deve iniciar a freagem através da resolução da equação (1). Para isto, todos os dados da via e da composição armazenados são carregados. Após a realização dos cálculos são armazenados como resultado o ponto e a velocidade de início de freagem. É importante notar que nesta etapa as variáveis elétricas não são computadas.

\subsection{Simulação objetivo}

O método utilizado para a resolução numérica da equação (1) foi, após alguns testes, o de Euler para uma primeira estimativa e o de Heun para encontrar uma solução mais precisa. O passo escolhido foi o de 1/16 segundos. Isto porque passos maiores produziram erros principalmente na parte da pré-simulação.

Basicamente, para cada passo, é realizada uma seqüência de cálculos. Primeiramente os cálculos referentes à dinâmica do movimento e em seguida os cálculos referentes à parte elétrica do trem.

Os resultados computados referentes à dinâmica do movimento são basicamente a velocidade, o espaço percorrido pelo trem, o tempo de percurso entre duas estações e a potência mecânica desenvolvida por esse trem. A força de aderência é obtida de acordo com a velocidade calculada no passo aplicada à equação (6) e então à equação (5).

A velocidade é utilizada também para se obter o esforço motor (ou esforço de freagem) e para calcular a resistência $R_{M P T}$. A posição do trem no espaço é utilizada para avaliar se existe ou não resistências do tipo acidentais ou alguma restrição de velocidade no trecho e o mais importante, para verificar se tal composição está ou não depois do ponto de freagem definido pela pré-simulação.

Com os resultados anteriormente obtidos pode-se ter o valor do torque, da velocidade de rotação no eixo do motor e da potência mecânica desenvolvida no eixo do motor conforme a equação (7), a equação (8) e a equação (9).

As variáveis elétricas dos motores são então calculadas. Para isto, é necessário saber, de acordo com a velocidade e com a potência desenvolvida pelo trem, em qual região de controle da característica dada de esforço motor em função da velocidade esta composição está. Conforme a região, o 
escorregamento ou a freqüência de escorregamento são mantidos constantes e o fluxo $\Phi_{A G}$ é então avaliado, como foi exposto no item 4.2. Faz-se então os cálculos das correntes no circuito equivalente.

A primeira corrente calculada é a corrente $I_{M}$ utilizando-se a equação (10). A corrente $I_{R}$ é então avaliada através da equação (11). Por último, a corrente $I_{S}$ é calculada através da equação (12). Vale notar que as constantes são calculadas no início da rotina a partir dos valores nominais.

É calculada então a tensão $U_{S}$ pela equação (14), o fator de potência neste passo e, finalmente, a corrente no pantógrafo ou coletor do terceiro trilho dada através da equação (16).

O passo é então terminado e um novo passo é iniciado, caso o trem não tenha chegado ao seu destino. $O$ espaço percorrido é então novamente avaliado para a verificação da existência ou não de resistências acidentais, limites de velocidade e para determinar se a composição está ou não dentro do espaço de freagem. Caso a composição esteja no espaço de freagem, os cálculos executados serão diferentes conforme o item 4.3 .

A velocidade calculada também é usada para o cálculo da resistência $R_{M P T}$ e para determinar em qual região de controle a composição se encontra. Caso a velocidade da composição seja a velocidade máxima permitida no trecho ou a velocidade máxima do trem, essa velocidade é mantida através de uma aceleração nula, significando um valor de $F_{\text {motor }}$ igual ao valor da soma dos esforços resistentes. Os demais cálculos são então repetidos.

\section{APLICAÇÃO}

O exemplo de aplicação aqui mostrado é a simulação de marcha de uma composição cuja locomotiva é acionada através de inversores de tensão e motores de indução do tipo gaiola e a rede de alimentação é em $3000 \mathrm{~V}$ corrente contínua, um dos sistemas de alimentação presentes no Brasil.

\subsection{Dados utilizados}

Tabela 1. Dados da Locomotiva.

\begin{tabular}{lr}
\hline \multicolumn{2}{c}{ Locomotiva } \\
\hline tipo: & $\mathrm{B}_{0}{ }^{\prime} \mathrm{B}_{0}{ }^{\prime}$ (quatro eixos \\
& motores divididos em dois \\
truques)
\end{tabular}

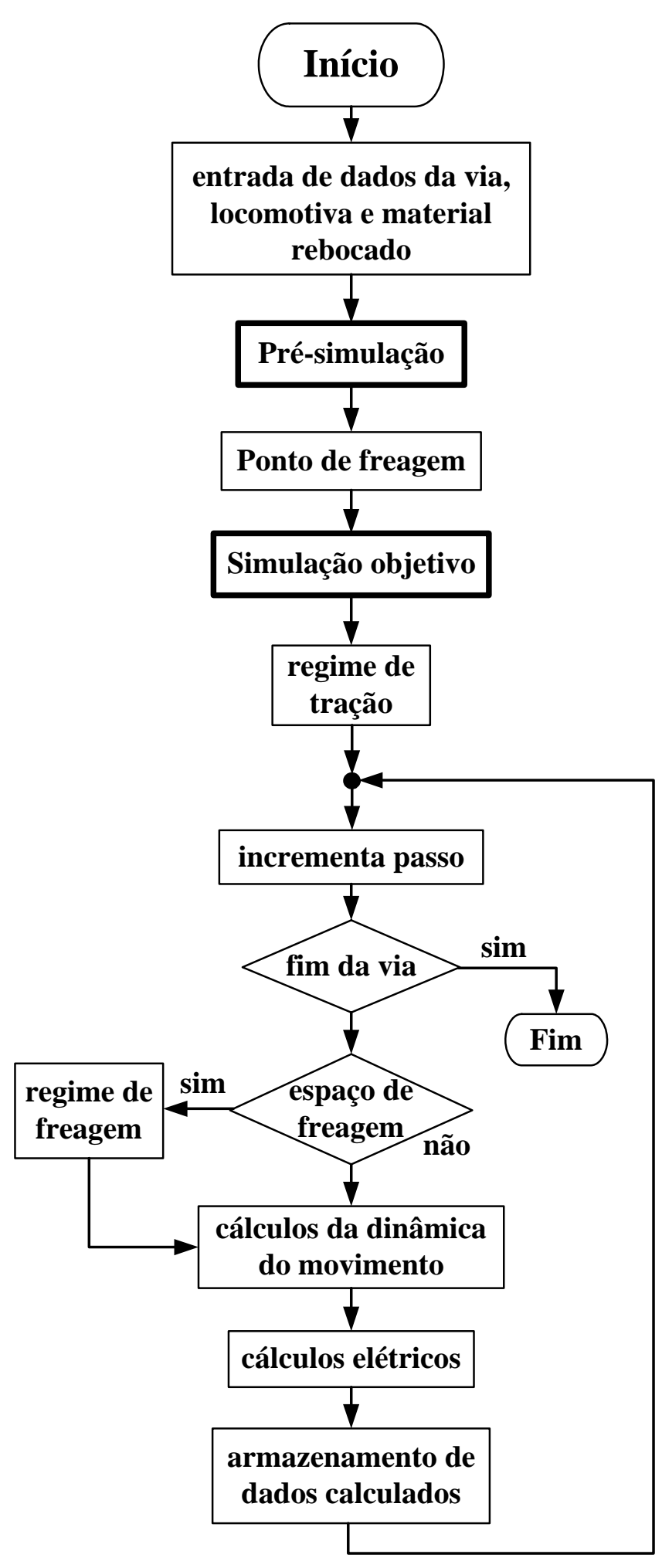

Figura 3 - Fluxograma das rotinas de cálculo. 
Tabela 2. Dados do Vagão.

\begin{tabular}{lr}
\hline \multicolumn{2}{c}{ Vagão } \\
\hline quantidade: & 13 \\
número de eixos: & 4 \\
massa: & $47,044 \mathrm{t}$ \\
seção transversal: & $12 \mathrm{~m}^{2}$ \\
\hline
\end{tabular}

Com estes dados, é possível calcular os coeficientes do trinômio da expressão de W. J. Davis Jr. (1926) para toda a composição conforme (Toledo et alii 1987). Deste modo a resistência normal total da composição em $\mathrm{kN}$ é:

$$
R_{M P T}=11,6490+0,09185 \cdot(3,6 \cdot v)+2,03732 \cdot 10^{-3} \cdot(3,6 \cdot v)^{2}
$$

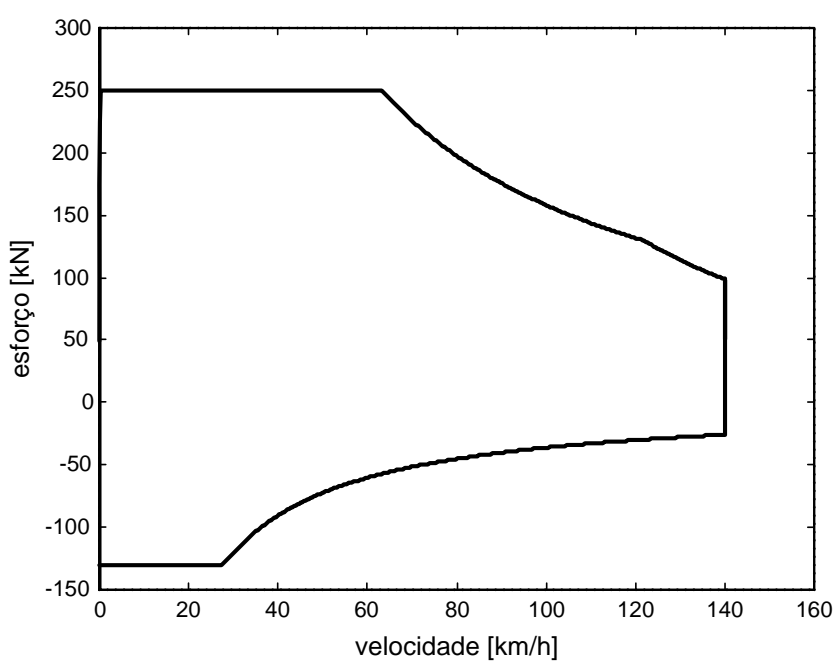

Figura 4 - Curva de esforço motor (tração / freagem) em função da velocidade.

Tabela 3. Dados da curva de esforço motor em função da velocidade (Figura 4).

\begin{tabular}{lr}
\hline potência desenvolvida em regime de tração: & 4400 \\
& $\mathrm{~kW}$ \\
potência desenvolvida em regime de freagem: & 1000 \\
& $\mathrm{~kW}$ \\
esforço de tração contínuo: & $250 \mathrm{kN}$ \\
esforço de freagem contínuo: & $130 \mathrm{kN}$ \\
\hline \multicolumn{1}{c}{ Tabela 4. Dados do Motor. } \\
\hline quantidade: & 4 \\
potência nominal: & $1105 \mathrm{~kW}$ \\
torque nominal: & $8,1 \mathrm{kN} . \mathrm{m}$ \\
número de pólos: & 6 \\
corrente nominal: & $364 \mathrm{~A}$ \\
tensão fase-fase: & $2190 \mathrm{~V}$ \\
velocidade de rotação nominal / & \\
freqüência: & $1300 \mathrm{rpm} / 66 \mathrm{~Hz}$ \\
&
\end{tabular}

Através da equação (9) pode-se ter o rendimento $\eta_{T}$ da transmissão que é de $99,55 \%$. Calculando-se o escorregamento nominal e a freqüência de escorregamento nominal tem-se, respectivamente, $1,5152 \%$ e $65 \mathrm{~Hz}$.

Antes da simulação, é necessário encontrar as variáveis elétricas restantes. Uma análise das potências em cada motor pelo circuito equivalente deve ser feita.

A potência desenvolvida pelo eixo do motor é a potência mecânica desenvolvida descontadas as perdas por atrito, ventilação, uma parte das perdas suplementares e as perdas no ferro conforme mostrado em Fitzgerald, Kingsley e Kusko (1975). Para a contabilização destas perdas, o parâmetro será o motor de indução utilizado em Largiadèr (1970) cuja potência nominal é de $1145 \mathrm{~kW}$ ou seja, bem próxima da potência nominal do motor utilizado pela locomotiva em questão. A soma destas perdas apresenta $2,215 \%$ de $P_{\text {motor }}$. Utilizando a mesma proporção, as perdas suplementares e as perdas no ferro nos motores da locomotiva em estudo representam $24,48 \mathrm{~kW}$. Desta forma, para as três fases tem-se uma potência mecânica desenvolvida de $1129,48 \mathrm{~kW}$.

Pela teoria do circuito equivalente do motor de indução apresentado em Fitzgerald, Kingsley e Kusko (1975), a potência dissipada nas barras do rotor e também no enrolamento do estator, considerando-se as perdas no estator e no rotor iguais, é uma fração $s$ de $1129,48 \mathrm{~kW}$. A fração (1-s) é a parte da potência que sai do entreferro e chega ao rotor. Contabilizando a perda no estator, a potência de entrada é de $1164,24 \mathrm{~kW}$ para as três fases.

Calculando através deste último resultado e também através dos dados do motor fornecidos, o fator de potência nominal é de $0,843\left(\varphi=32,54^{\circ}\right)$.

A corrente por fase do estator pode então ser escrita na sua forma fasorial, assumindo a fase da tensão no estator igual a $0^{\circ}$ :

$\dot{I}_{S}=364 \angle \varphi^{\mathrm{o}}=364 \angle 32,54^{\mathrm{o}}=306,8577+j \cdot 195,7916$

Pode-se notar que a corrente $I_{S}$ na equação (18) é composta da corrente $I_{M}$ atrasada de $90^{\circ}$ da corrente $I_{R}$. Assim, o valor nominal de $I_{R}$ é de 306,8577 A e o valor nominal de $I_{M}$ é de $195,7916 \mathrm{~A}$.

\subsection{Resultados obtidos}

O caso aqui mostrado é uma via de $25000 \mathrm{~m}$ dividida em cinco tipos de perfil. O primeiro perfil tem o comprimento de $5000 \mathrm{~m}$ e é plano. O perfil seguinte tem o comprimento de $5000 \mathrm{~m}$ e há um declive de $2 \%$. O terceiro perfil também de $5000 \mathrm{~m}$ é uma curva de raio igual $3183 \mathrm{~m}$. O 
quarto, de comprimento igual aos anteriores, é um aclive de $5 \%$. Por último, tem-se novamente um perfil plano.

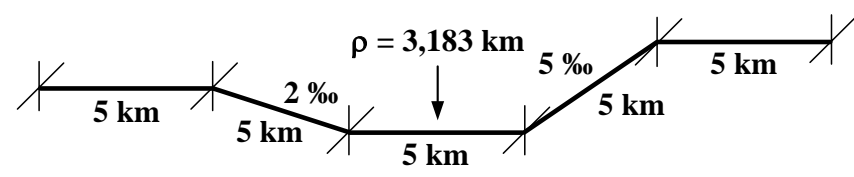

Figura 5 - Perfil da via utilizada.

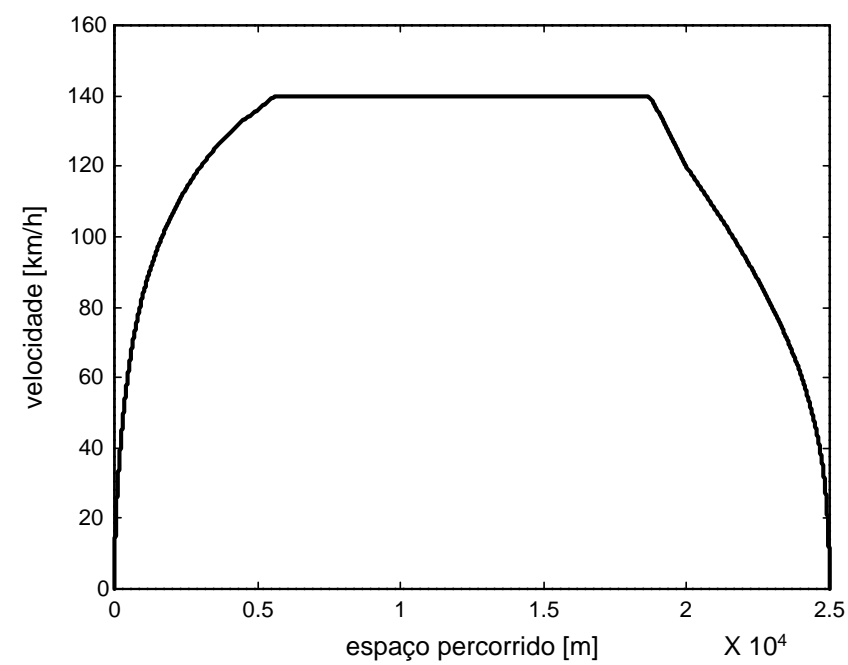

Figura 6 - Velocidade do trem em função do espaço percorrido.

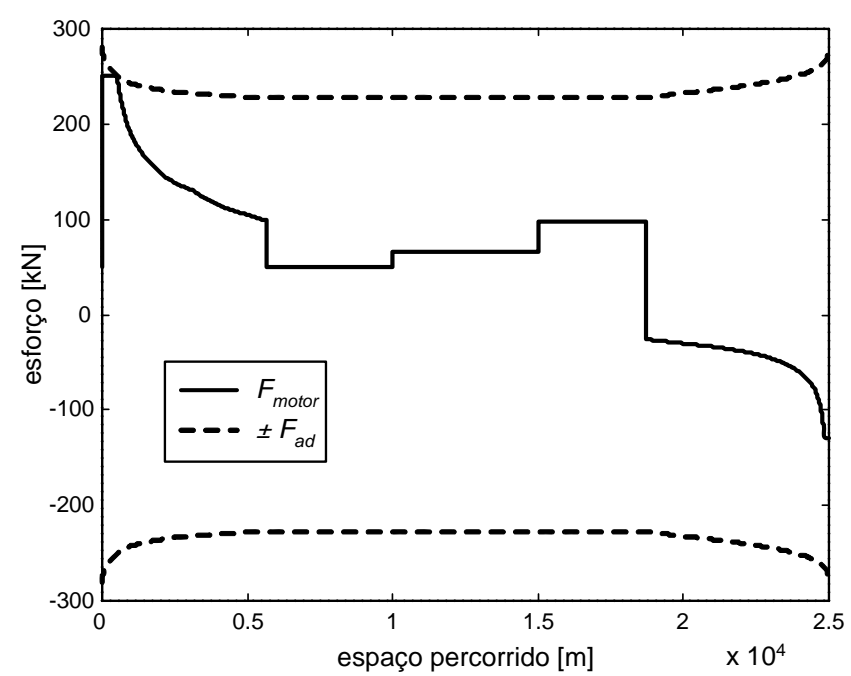

Figura 7 - Esforço motor e força de aderência em função do espaço percorrido.

Pela Figura 7 pode-se notar que no período de velocidade constante, o esforço motor exercido é aquele necessário para que apenas as resistências normais e acidentais sejam vencidas.

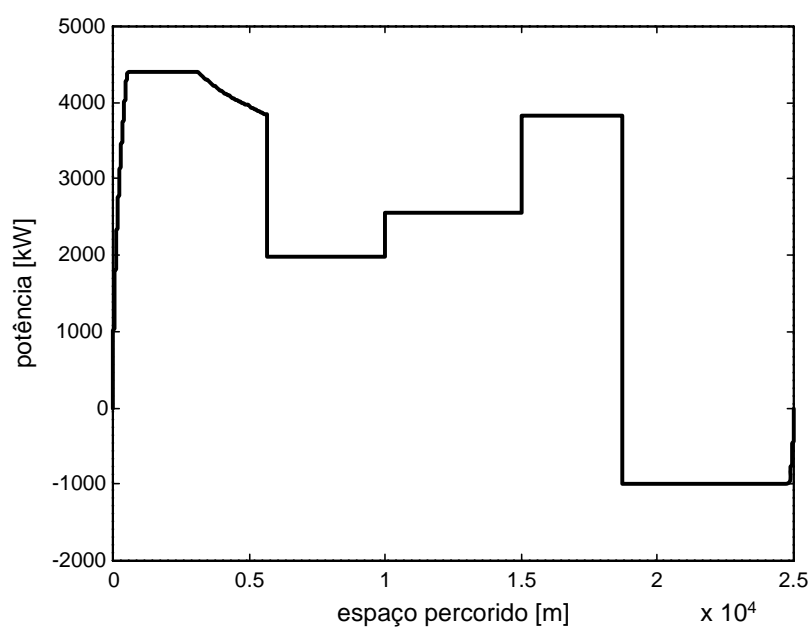

Figura 8 - Potência $P_{U}$ em função do espaço percorrido.

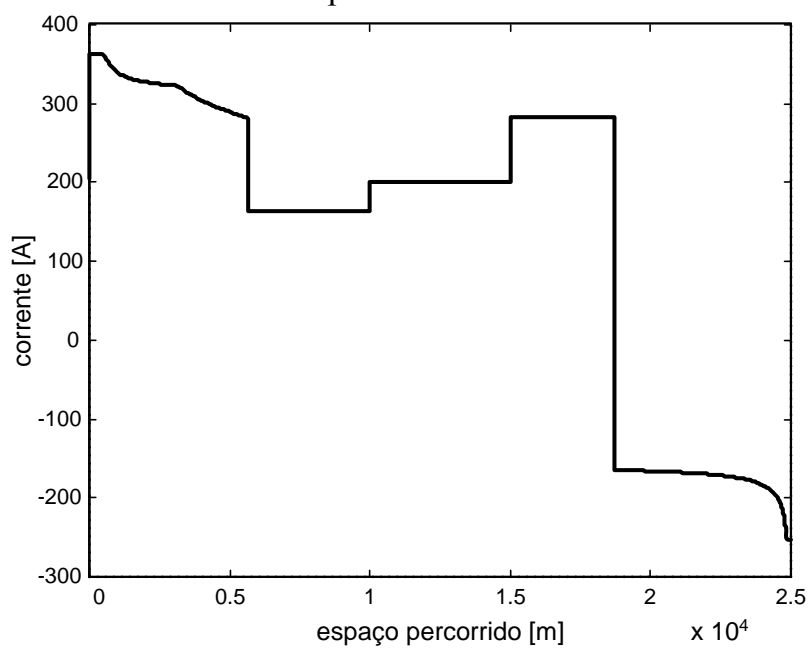

Figura 9 - Corrente $I_{S}$ em cada motor em função do espaço

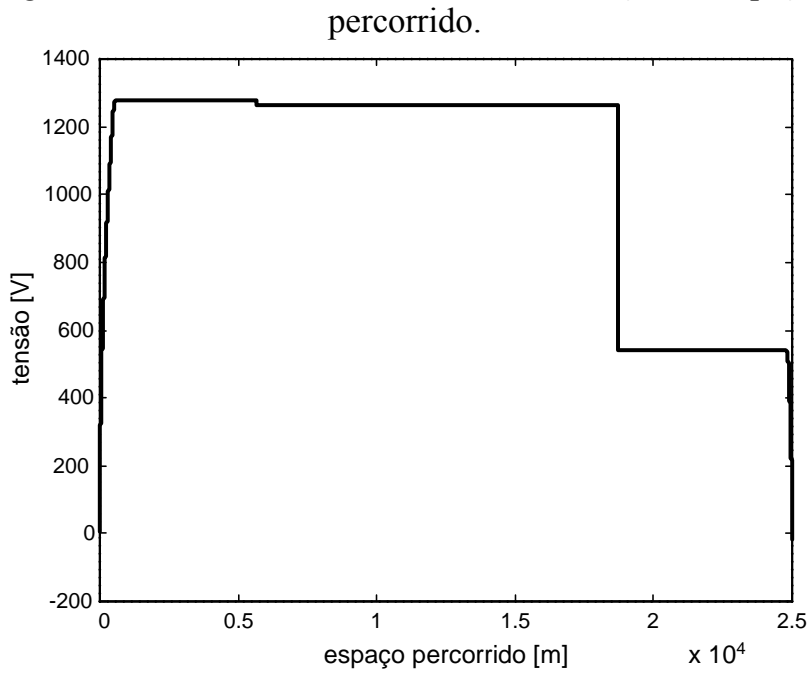

Figura 10 - Tensão $U_{S}$ por motor em função do espaço percorrido. 


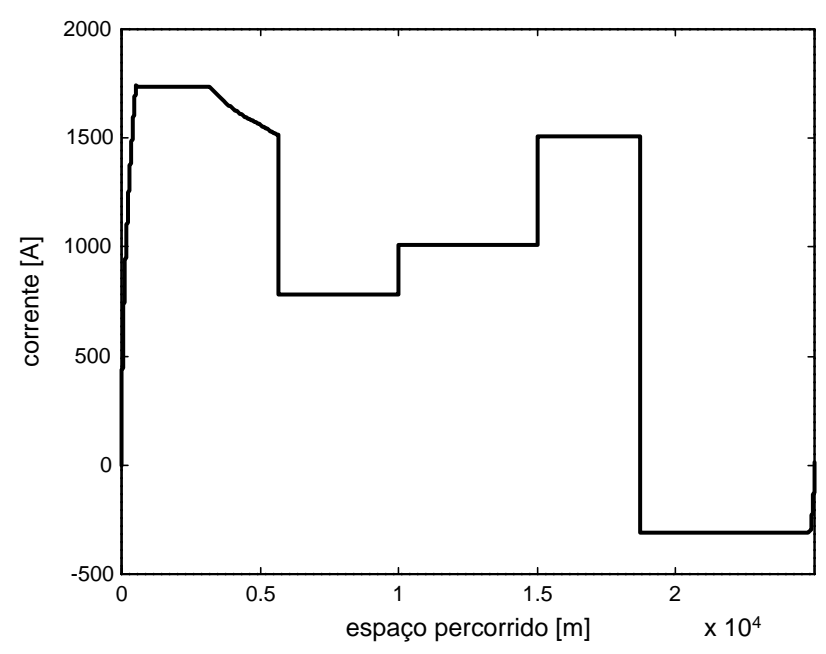

Figura 11 - Corrente $I_{d}$ em função do espaço percorrido.

É importante notar que na Figura 11, a corrente negativa produzida durante o período de freagem pode tanto ser regenerada e consumida por uma outra composição, se os equipamentos da locomotiva e da rede permitirem, quanto ser consumida por um resistor de freagem. Apenas uma avaliação do sistema completo com todos os trens pode definir o destino desta corrente gerada.

Através da leitura do artigo e da análise dos resultados percebe-se que para chegar ao valor da corrente captada pelo pantógrafo ou coletor do terceiro trilho um cálculo trabalhoso deve ser feito. Porém, os resultados mostram que o formato da curva da potência desenvolvida pelo veículo e o formato da curva da corrente captada são semelhantes. Isto pode sugerir uma proporção linear entre as duas grandezas. É interessante então desenvolver este ponto.

A potência $P_{\text {in }}$ pode ser definida como:

$$
P_{\text {in }}=U_{S} \cdot I_{S} \cdot \cos (\varphi) \cdot 10^{-3}
$$

E o rendimento do motor pode ser dado através de:

$$
\eta_{m}=\frac{P_{m o t o r}}{3 \cdot P_{i n}}
$$

Aplicando a equação (19) e a equação (20) na equação (16) e substituindo $P_{\text {motor }}$ de acordo com a equação (9), tem-se:

$$
I_{d}=\frac{P_{U}}{U_{d} \cdot \eta_{m} \cdot \eta_{T} \cdot 10^{-3}}
$$

Comparando os resultados obtidos pelas simulações com a aplicação direta da equação (21), nota-se que as correntes fornecidas através desta expressão simplificada possuem um valor menor do que as calculadas através de simulações durante o regime de tração e um módulo maior do que as correntes calculadas durante o regime de freagem.

O erro entre a corrente obtida através da simulação e a corrente dada aplicando a equação (21) é maior em velocidades mais baixas, chegando a uma diferença de 14,7 A $(0,85 \%)$ para a região de potência constante no regime de tração.

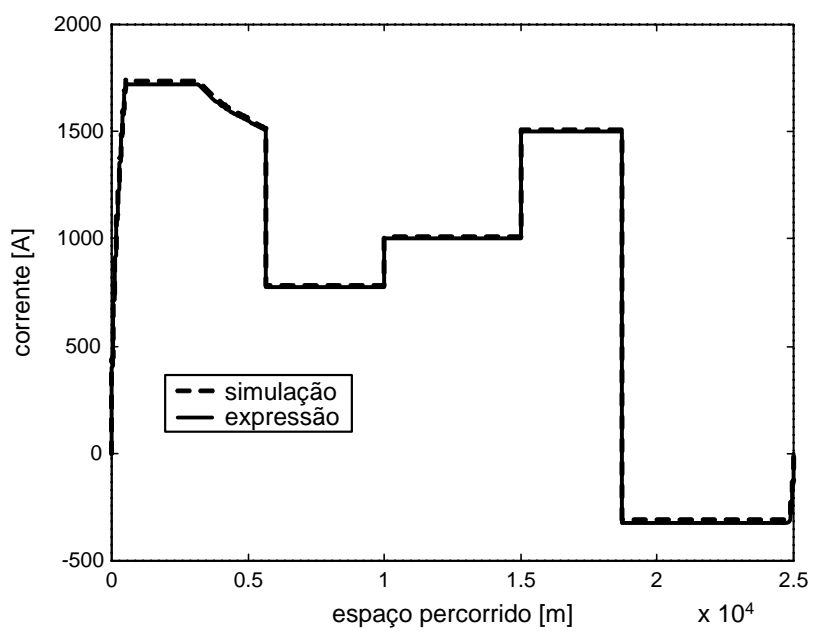

Figura 12 - Comparação de resultados.

\section{CONSIDERAÇÕES FINAIS}

Através da leitura do artigo pôde-se perceber a importância da simulação ferroviária. Prever o comportamento de um trem ao longo de uma via entre duas estações adjacentes é uma tarefa laboriosa, o que torna o uso de ferramentas computacionais indispensável.

Além das variáveis mecânicas como a velocidade e a potência do trem, é interessante conhecer também a corrente consumida por este trem durante o seu percurso. Para isso, o acoplamento entre as variáveis mecânicas e elétricas através de um modelo do motor de indução e do inversor levando em consideração o controle foi feito.

Os resultados mostraram que todo o equacionamento do motor de indução para o cálculo das correntes de estator, rotor e magnetização, para o cálculo da tensão aplicada ao estator bem como a sua freqüência durante a operação do trem pode ser resumido em uma única expressão simplificada. Esta simplificação é de grande valia uma vez que as grandezas do circuito equivalente do motor de indução envolvidas no cálculo da corrente captada não são normalmente fornecidas com os dados dos trens ou encontradas em catálogos, sendo necessária a aplicação de um modelo para encontrá-los, como foi feito neste trabalho.

Justamente por utilizar dados de catálogo, tal simplificação é também bastante útil para cálculos rápidos em um dado instante de tempo envolvendo a corrente captada pelo trem. 


\section{REFERÊNCIAS BIBLIOGRAFICAS}

Courtois, C. et alii, (1998). Traction électrique ferroviaire. 1.ed. Paris: Techniques de l'ingénieur.

Davis Jr., W. J. (1926). The tractive resistance of electric locomotives and cars. General Electric Review, v.29, n. 10 , p. $685-707$.

Filipović, Ž. (1995). Elektrische Bahnen Grundlagen Triebfahrzeuge Stromversorgung. 3.ed. Heidelberg: Springer.

Fitzgerald, A. E., C. Kingsley Jr. e A. Kusko (1975). Máquinas elétricas. 1.ed. São Paulo: Makron Books.

Hill, R. J. (1994). Electric railway traction Part 2 Traction drives with three-phase induction motors. Power Engineering Journal (June), p.143-152.

Kaller, R. et J. -M. Allenbach (1995). Traction eléctrique. 1.ed. Lausanne: Presses Universitaires Romandes.

Langsdorf, A. S. (1981). Sec.8-21: The asynchronous or induction generator. Theory of alternating current machinery, p. 354-362 New Delhi: Tata McGraw-Hill.

Largiadèr, H. (1970). Design aspects of induction motors for traction applications with supply through static frequency changers. Brown Boveri Review, v.57, n. 4, p. 152-167.

Martins, R. W. C. (1986). Sistemas de tração elétrica: análise e dimensionamento. Dissertação (Mestrado) Escola Politécnica, Universidade de São Paulo, São Paulo.

Mohan, N., T. Undeland and W. P. Robbins (1995). Power electronics converters, applications and design. 2.ed. New York: John Wiley \& Sons.

Pires, C. L. (2002). Simulação de marcha de composição ferroviária acionada por motores de indução e PWM. Dissertação (Mestrado) - Escola Politécnica, Universidade de São Paulo, São Paulo.

Plette, D. L. and A. B. Plunkett (1977). Inverter-induction motor drive for transit cars. IEEE Transactions on Industry Applications, v. IA-13, n. 1, p. 26-37.

Steimel, A. (1998). Control of the induction machine in traction. Elektrische Bahnen, v. 96, n. 12, p. 361-369.

Toledo, E. D. et alii, (1987). Tração elétrica. 1.ed. São Paulo: Nobel. 